\title{
ON THE SOLUTIONS OF A SYSTEM OF $(2 p+1)$ DIFFERENCE EQUATIONS OF HIGHER ORDER
}

\author{
A. KHELIFA, Y. HALIM, AND M. BERKAL
}

Received 17 June, 2020

\begin{abstract}
In this paper we represent the well-defined solutions of the system of the higher-order rational difference equations

$$
x_{n+1}^{(j)}=\frac{1+2 x_{n-k}^{(j+1) \bmod (2 p+1)}}{3+x_{n-k}^{(j+1) \bmod (2 p+1)}}, \quad n, k, p \in \mathbb{N}_{0}
$$

in terms of Fibonacci and Lucas sequences, where the initial values $x_{-k}^{(j)}, x_{-k+1}^{(j)}, \ldots, x_{-1}^{(j)}$ and $x_{0}^{(j)}$, $j=1,2, \ldots, 2 p+1$, do not equal -3 . Some theoretical explanations related to the representation for the general solution are also given.

2010 Mathematics Subject Classification: 39A10; 40A05

Keywords: Fibonacci sequence, Lucas sequence, system of difference equations, representation of solutions
\end{abstract}

\section{INTRODUCTION}

The seniority, richness and the appreciable flexibility of use, have allowed difference equations to be an attractive subject in recent times among researchers and scientists from different disciplines. Difference equations and system of difference equations have been applied in diverse mathematical models in biology, economics, genetics, population dynamics, medicine, and other fields (see $[4,8,17]$ ).

Solving system of difference equations in closed-form has attracted the attention of many authors, (see, for example [1-3,5-7, 9-16,18,21-23] and the references therein).

It is a well-known fact that the Fibonacci sequence defined as follows

$$
F_{n+1}=F_{n}+F_{n-1}, \quad n \in \mathbb{N},
$$

where $F_{0}=0$ and $F_{1}=1$. The solution of equation (1.1) is given by the formula

$$
F_{n}=\frac{\alpha^{n}-\beta^{n}}{\alpha-\beta}
$$

The work was supported by DGRSDT-MESRS (DZ). 
which is called the Binet formula of the Fibonacci numbers, where

$$
\alpha=\frac{1+\sqrt{5}}{2} \text { (the so }- \text { called golden number), } \beta=\frac{1-\sqrt{5}}{2} \text {. }
$$

One can easily verify that

$$
\lim _{n \rightarrow+\infty} \frac{F_{n+r}}{F_{n}}=\alpha^{r}, \quad n, r \in \mathbb{N} .
$$

Also, the Lucas sequence has the same recursive relationship as the Fibonacci sequence,

$$
L_{n+1}=L_{n}+L_{n-1}, \quad n \in \mathbb{N},
$$

but with different initial conditions, $L_{0}=2$ and $L_{1}=1$. The first few terms of the recurrence sequence are $2,1,3,4,7,11,18,29,47,76, \ldots$. The Binet's formula for this recurrence sequence can easily be obtained and is given by

$$
L_{n}=\alpha^{n}+\beta^{n},
$$

where $\alpha$ and $\beta$ are the two numbers mentioned in (1.3), and we have also

$$
\lim _{n \rightarrow+\infty} \frac{L_{n+r}}{L_{n}}=\alpha^{r}, \quad n, r \in \mathbb{N} .
$$

Khelifa et al. in [19] gave some theoretical explanations related to the representation for the general solution of the system of three higher-order rational difference equations

$$
x_{n+1}=\frac{1+2 y_{n-k}}{3+y_{n-k}}, \quad y_{n+1}=\frac{1+2 z_{n-k}}{3+z_{n-k}}, \quad z_{n+1}=\frac{1+2 x_{n-k}}{3+x_{n-k}}, \quad n, k \in \mathbb{N}_{0} .
$$

Motivated by the paper [19], we represents the well-defined solutions of the system of $(2 p+1)$ higher-order rational difference equations

$$
x_{n+1}^{(j)}=\frac{1+2 x_{n-k}^{(j+1) \bmod (2 p+1)}}{3+x_{n-k}^{(j+1) \bmod (2 p+1)}}, \quad n, k, p \in \mathbb{N}_{0}, j=1,2, \ldots, 2 p+1 .
$$

Clearly if take $p=1$ in the system (1.9) we get the system (1.8). So our results generalizes the results obtained in [19].

\section{ON THE SYSTEM OF FIRST ORDER DIFFERENCE EQUATIONS}

In this section, to give a closed form for the well defined solutions of the system (1.9) we consider the system of $2 p+1$ difference equations of first order

$$
x_{n+1}^{(1)}=\frac{1+2 x_{n}^{(2)}}{3+x_{n}^{(2)}}, \quad x_{n+1}^{(2)}=\frac{1+2 x_{n}^{(3)}}{3+x_{n}^{(3)}}, \quad \ldots, \quad x_{n+1}^{(2 p+1)}=\frac{1+2 x_{n}^{(1)}}{3+x_{n}^{(1)}}, \quad n, p \in \mathbb{N}_{0} .
$$


We replace $x_{n+1}^{(2 p+1)}$ in the equation $x_{n+1}^{(2 p)}=\frac{1+2 x_{n}^{(2 p+1)}}{3+x_{n}^{(2 p+1)}}$, we get

$$
x_{n+1}^{(2 p)}=\frac{F_{2}+F_{1} x_{n-1}^{(1)}}{F_{3}+F_{2} x_{n-1}^{(1)}}, \quad n \geq 1 .
$$

Similarly, we replace $x_{n+1}^{(2 p)}$ in the equation $x_{n+1}^{(2 p-1)}=\frac{1+2 x_{n}^{(2 p)}}{3+x_{n}^{(2 p)}}$, we get

$$
x_{n+1}^{(2 p-1)}=\frac{L_{3}+L_{2} x_{n-2}^{(1)}}{L_{4}+L_{3} x_{n-2}^{(1)}} \quad n \geq 2 .
$$

By induction we get

$$
\begin{array}{ll}
x_{n+1}^{(2)}=\frac{F_{2 p}+F_{2 p-1} x_{n-2 p+1}^{(1)}}{F_{2 p+1}+F_{2 p} x_{n-2 p+1}^{(1)}}, \quad n \geq(2 p-1), \\
x_{n+1}^{(1)}=\frac{L_{2 p+1}+L_{2 p} x_{n-2 p}^{(1)}}{L_{2 p+2}+L_{2 p+1} x_{n-2 p}^{(1)}}, \quad n \geq 2 p .
\end{array}
$$

So, the system (2.1) can be written as the following equation

$$
x_{n+1}=\frac{L_{2 p+1}+L_{2 p} x_{n-2 p}}{L_{2 p+2}+L_{2 p+1} x_{n-2 p}} \quad n \geq 2 p .
$$

Let

$$
{ }^{(j)} x_{n}=x_{(2 p+1) n+j}, \quad n \in \mathbb{N}_{0}
$$

where $j \in\{0,1,2, \cdots, 2 p\}$.

Using notation (2.3), we can write (2.2) as

$$
{ }^{(j)} x_{n+1}=\frac{L_{2 p+1}+L_{2 p}{ }^{(j)} x_{n}}{L_{2 p+2}+L_{2 p+1}{ }^{(j)} x_{n}}, \quad n \in \mathbb{N}
$$

for each $j \in\{0,1,2, \cdots, 2 p\}$.

Now consider the equation

$$
y_{n+1}=\frac{L_{2 p+1}+L_{2 p} y_{n}}{L_{2 p+2}+L_{2 p+1} y_{n}} \quad n \in \mathbb{N}_{0}
$$

Using the change of variables

$$
y_{n}=\frac{1}{L_{2 p+1}}\left(w_{n}-L_{2 p+2}\right), \quad n \in \mathbb{N}_{0}
$$

we can write (2.5) as

$$
w_{n+1}=\frac{\left(L_{2 p}+L_{2 p+2}\right) w_{n}-5}{w_{n}}, \quad n \in \mathbb{N}_{0}
$$


In the following result, we solve in a closed form the equation (2.8) in terms of the sequences $\left(F_{n}\right)_{n=0}^{+\infty}$ and $\left(L_{n}\right)_{n=0}^{+\infty}$. The obtained formula will be very useful to get the formula of the solutions of system (1.9).

Lemma 1. Consider the linear difference equation

$$
z_{n+1}-5 F_{2 p+1} z_{n}+5 z_{n-1}=0, \quad n \in \mathbb{N}_{0},
$$

with initial conditions $z_{-1}, z_{0} \in \mathbb{R}$. Then all solutions of equation (2.8) will be written under the form

$$
z_{n}=\left(\frac{\sqrt{5}^{n}}{L_{2 p+1}}\right)\left[\sqrt{5} z_{-1} N_{(2 p+1) n}-z_{0} N_{(2 p+1)(n+1)}\right],
$$

where

$$
N_{(2 p+1) n}=\left(\alpha^{(2 p+1) n}-(-1)^{n} \beta^{(2 p+1) n}\right), \quad \text { with } \quad \alpha=\frac{1+\sqrt{5}}{2}, \beta=\frac{1-\sqrt{5}}{2} .
$$

So,

$$
N_{(2 p+1) n}=\left\{\begin{array}{cl}
\sqrt{5} F_{(2 p+1) n}, & \text { if } n \text { even, } \\
L_{(2 p+1) n}, & \text { if } n \text { odd },
\end{array}\right.
$$

where $\left(F_{n}\right)_{n=0}^{+\infty}$ is the Fibonacci sequence and $\left(L_{n}\right)_{n=0}^{+\infty}$ is the Lucas sequence.

Proof. As it is well-known, the equation

$$
z_{n+1}-5 F_{2 p+1} z_{n}+5 z_{n-1}=0, \quad n \in \mathbb{N}_{0},
$$

(the homogeneous linear second order difference equation with constant coefficients), where $z_{0}, z_{-1} \in \mathbb{R}$, is usually solved by using the characteristic roots $\lambda_{1}$ and $\lambda_{2}$ of the characteristic polynomial $\lambda^{2}-5 F_{2 p+1} \lambda+5$. So

$$
\lambda_{1}=\frac{5 F_{2 p+1}+\sqrt{5} L_{2 p+1}}{2}, \quad \lambda_{2}=\frac{5 F_{2 p+1}-\sqrt{5} L_{2 p+1}}{2}
$$

and the formula of general solution is

$$
x_{n}=c_{1} \lambda_{1}^{n}+c_{2} \lambda_{2}^{n} .
$$

The characteristic roots $\lambda_{1}$ and $\lambda_{2}$ check the following relationships

$$
\begin{aligned}
& \lambda_{1}=\frac{5 F_{2 p+1}+\sqrt{5} L_{2 p+1}}{2}=\sqrt{5}\left(\frac{L_{2 p+1}+\sqrt{5} F_{2 p+1}}{2}\right)=\sqrt{5} \alpha^{2 p+1}, \\
& \lambda_{2}=\frac{5 F_{2 p+1}-\sqrt{5} L_{2 p+1}}{2}=-\sqrt{5}\left(\frac{L_{2 p+1}-\sqrt{5} F_{2 p+1}}{2}\right)=-\sqrt{5} \beta^{2 p+1} .
\end{aligned}
$$

Using the initial conditions $z_{0}$ and $z_{-1}$ with some calculations we get

$$
c_{1}=-\frac{\sqrt{5}}{L_{2 p+1}}\left(z_{-1}-\frac{z_{0}}{5} \lambda_{1}\right),
$$




$$
c_{2}=-\frac{\sqrt{5}}{L_{2 p+1}}\left(\frac{z_{0}}{5} \lambda_{2}-z_{-1}\right)
$$

So,

$$
\begin{aligned}
z_{n}= & \left(-\frac{\sqrt{5}}{L_{2 p+1}}\left(z_{-1}-\frac{z_{0}}{5} \lambda_{1}\right)\right) \lambda_{1}^{n}+\left(-\frac{\sqrt{5}}{L_{2 p+1}}\left(\frac{z_{0}}{5} \lambda_{2}-z_{-1}\right)\right) \lambda_{2}^{n} \\
= & -\frac{\sqrt{5}}{L_{2 p+1}}\left(z_{-1}\left[\lambda_{1}^{n}-\lambda_{2}^{n}\right]-\frac{z_{0}}{5}\left[\lambda_{1}^{n+1}-\lambda_{2}^{n+1}\right]\right) \\
= & -\frac{\sqrt{5}}{L_{2 p+1}}\left(z_{-1}(\sqrt{5})^{n}\left[\alpha^{(2 p+1) n}-(-1)^{n} \beta^{(2 p+1) n}\right]\right. \\
& \left.-\frac{z_{0}(\sqrt{5})^{n+1}}{(\sqrt{5})^{2}}\left[\alpha^{(2 p+1)(n+1)}-(-1)^{n+1} \beta^{(2 p+1)(n+1)}\right]\right),
\end{aligned}
$$

putting

$$
N_{(2 p+1) n}=\left(\alpha^{(2 p+1) n}-(-1)^{n} \beta^{(2 p+1) n}\right),
$$

it is obtained that the general solution of equation (2.8) is

$$
\left.z_{n}=-\frac{(\sqrt{5})^{n}}{L_{2 p+1}}\left[z_{-1} \sqrt{5} N_{(} 2 p+1\right) n-z_{0} N_{(2 p+1)(n+1)}\right] .
$$

The lemma is proved.

Through an analytical approach we put

$$
w_{n}=\frac{z_{n}}{z_{n-1}},
$$

which reduces equation (2.7) to the following one

$$
z_{n+1}=5 F_{2 p+1} z_{n}-5 z_{n-1}
$$

So, from Lemma (1) we get

$$
z_{n}=\left(\frac{\sqrt{5}^{n}}{L_{2 p+1}}\right)\left[\sqrt{5} z_{-1} N_{(2 p+1) n}-z_{0} N_{(2 p+1)(n+1)}\right],
$$

with

$$
N_{(2 p+1) n}=\left\{\begin{array}{cl}
\sqrt{5} F_{(2 p+1) n}, & \text { if } n \text { even, } \\
L_{(2 p+1) n}, & \text { if } n \text { odd }
\end{array}\right.
$$

where $\left(F_{n}\right)_{n=0}^{+\infty}$ is the Fibonacci sequence and $\left(L_{n}\right)_{n=0}^{+\infty}$ is the Lucas sequence. 
By formulas (2.12) and (2.14), it follows that the general solution of equation (2.7) is

$$
\left\{\begin{array}{l}
w_{2 n}=\frac{5 F_{2(2 p+1) n}-w_{0} L_{(2 p+1)(2 n+1)}}{L_{(2 p+1)(2 n-1)}-w_{0} F_{2(2 p+1) n}} \\
w_{2 n+1}=\frac{5 L_{(2 p+1)(2 n+1)}-5 w_{0} F_{2(2 p+1)(n+1)}}{5 F_{2(2 p+1) n}-w_{0} L_{(2 p+1)(2 n+1)}} .
\end{array}\right.
$$

From all above mentioned the following theorem holds.

Theorem 1. Let $\left\{y_{n}\right\}_{n \geq 0} b$ a well-defined solution of the equation (2.5). Then, for $n=2,3, \ldots$,

$$
\left\{\begin{array}{l}
y_{2 n}=\frac{F_{2(2 p+1) n}+F_{2(2 p+1) n-1} y_{0}}{F_{2(2 p+1) n+1}+F_{2(2 p+1) n} y_{0}} \\
y_{2 n+1}=\frac{L_{2(2 p+1) n+(2 p+1)}+L_{2(2 p+1) n+2 p} y_{0}}{L_{2(2 p+1) n+(2 p+2)}+L_{2(2 p+1) n+(2 p+1)} y_{0}}
\end{array}\right.
$$

where $\left(L_{n}\right)_{n=0}^{+\infty}$ is the Lucas sequence and $\left(F_{n}\right)_{n=0}^{+\infty}$ is the Fibonacci sequence.

Proof. According to the change of variable (2.6), and using the following equalities (see [20])

$$
\begin{aligned}
L_{2 p+1} F_{2(2 n+1) n+1} & =L_{2 p+2} F_{2(2 p+1) n-1}-L_{2(2 p+1) n-(2 p+2)} \\
L_{2 p+1} L_{2(2 p+1) n+(2 p+2)} & =L_{2 p+1} L_{2(2 p+1) n+(2 p+1)}-5 F_{2(2 p+1) n} \\
L_{2 p+1} F_{2(2 p+1) n-1} & =L_{2(2 p+1) n+(2 p+1)}-L_{2 p+2} F_{2(2 p+1) n} \\
L_{2 p+1} L_{2(2 p+1) n-(2 p+2)} & =5 F_{2(2 p+1) n}-L_{2 p+2} L_{2(2 p+1) n-(2 p+1)}
\end{aligned}
$$

we obtain

$$
\begin{aligned}
y_{2 n}= & \frac{1}{L_{2 p+1}}\left(w_{2 n}-L_{2 p+2}\right) \\
= & \frac{1}{L_{2 p+1}}\left(\frac{\left(5 F_{2(2 p+1) n}-L_{2 p+2} L_{2(2 p+1) n-(2 p+1)}\right)}{L_{2(2 n+1) n-(2 n+1)}-w_{0} F_{2(2 n+1) n}}\right) \\
& +\frac{1}{L_{2 p+1}}\left(\frac{+w_{0}\left(L_{2 p+2} F_{2(2 p+1) n}-L_{2(2 p+1) n+(2 p+1)}\right)}{L_{2(2 n+1) n-(2 n+1)}-w_{0} F_{2(2 n+1) n}}\right) \\
= & \frac{1}{L_{2 p+1}}\left(\frac{L_{2 p+1} L_{2(2 p+1) n-(2 p+2)}-L_{2 p+1} w_{0} F_{2(2 p+1) n-1}}{L_{2(2 p+1) n-(2 p+1)}-w_{0} F_{2(2 p+1) n}}\right) \\
= & \frac{\left(L_{2(2 p+1) n-(2 p+2)}-L_{2 p+2} F_{2(2 p+1) n-1}\right)-L_{2 p+1} y_{0} F_{2(2 p+1) n-1}}{\left(L_{2(2 p+1) n-(2 p+1)}-L_{2 p+2} F_{2(2 p+1) n}\right)-L_{2 p+1} y_{0} F_{2(2 p+1) n}} \\
= & \frac{-L_{2 p+1} F_{2(2 p+1) n}-L_{2 p+1} y_{0} F_{2(2 p+1) n-1}}{-L_{2 p+1} F_{2(2 p+1) n+1}-L_{2 p+1} y_{0} F_{2(2 p+1) n}} .
\end{aligned}
$$


So

Similarly

$$
y_{2 n}=\frac{F_{2(2 p+1) n}+y_{0} F_{2(2 p+1) n-1}}{F_{2(2 p+1) n+1}+y_{0} F_{2(2 p+1) n}} .
$$

$$
\begin{aligned}
y_{2 n+1}= & \frac{1}{L_{2 p+1}}\left(w_{2 n+1}-L_{2 p+2}\right) \\
= & \frac{1}{L_{2 p+1}}\left(\frac{5\left(L_{2(2 p+1) n+(2 p+1)}-L_{2 p+2} F_{2(2 p+1) n}\right)}{5 F_{2(2 p+1) n}-w_{0} L_{2(2 p+1) n+(2 p+1)}}\right) \\
& +\frac{1}{L_{2 p+1}}\left(\frac{-w_{0}\left(5 F_{2(2 p+1) n+(2 p+1)}-7 L_{2(2 p+1) n+2(2 p+1)}\right)}{5 F_{2(2 p+1) n}-w_{0} L_{2(2 p+1) n+(2 p+1)}}\right) \\
= & \frac{L_{2 p+1}}{L_{2 p+1}}\left(\frac{5 F_{2(2 p+1) n-1}-w_{0} L_{2(2 p+1)(n+1)-(2 p+2)}}{5 F_{2(2 p+1) n}-w_{0} L_{2(2 p+1) n+(2 p+1)}}\right) \\
= & \frac{\left(5 F_{2(2 p+1) n-1}-L_{2 p+2} L_{2(2 p+1) n+2 p}\right)-L_{2 p+1} y_{0} L_{2(2 p+1) n+2 p}}{\left(5 F_{2(2 p+1) n}-L_{2 p+1} L_{2(2 p+1) n+(2 p+1)}\right)-L_{2 p+1} y_{0} L_{2(2 p+1) n+(2 p+1)}} \\
= & \frac{-L_{2 p+1}}{-L_{2 p+1}}\left(\frac{L_{2(2 p+1) n+(2 p+1)}+y_{0} L_{2(2 p+1) n+2 p}}{L_{2(2 p+1) n+(2 p+2)}+y_{0} L_{2(2 p+1) n+(2 p+1)}}\right) .
\end{aligned}
$$

So

$$
y_{2 n+1}=\frac{L_{2(2 p+1) n+(2 p+1)}+y_{0} L_{2(2 p+1) n+2 p}}{L_{2(2 p+1) n+(2 p+2)}+y_{0} L_{2(2 p+1) n+(2 p+1)}} .
$$

From Theorem (1), the solution of equation (2.4) given by

$$
\left\{\begin{array}{l}
{ }^{(j)} x_{2 n}=\frac{F_{2(2 p+1) n}+F_{2(2 p+1) n-1}{ }^{(j)} x_{0}}{F_{2(2 p+1) n+1}+F_{2(2 p+1) n}{ }^{(j)} x_{0}} \\
{ }^{(j)} x_{2 n+1}=\frac{L_{2(2 p+1) n+(2 p+1)}+L_{2(2 p+1) n+2 p}{ }^{(j)} x_{0}}{L_{2(2 p+1) n+(2 p+2)}+L_{2(2 p+1) n+(2 p+1)}{ }^{(j)} x_{0}} .
\end{array}\right.
$$

By using (2.3) the following corollary is easily obtained from Theorem (1).

Corollary 1. Let $\left\{x_{n}\right\}_{n \geq 0}$ be a well-defined solution of (2.2). Then, for, $n \geq 2 p$

$$
\left\{\begin{array}{l}
x_{(2 p+1)(2 n)+j}=\frac{F_{2(2 p+1) n}+F_{2(2 p+1) n-1} x_{j}}{F_{2(2 p+1) n+1}+F_{2(2 p+1) n} x_{j}}, \\
x_{(2 p+1)(2 n+1)+j}=\frac{L_{2(2 p+1) n+(2 p+1)}+L_{2(2 p+1) n+2 p} x_{j}}{L_{2(2 p+1) n+(2 p+2)}+L_{2(2 p+1) n+(2 p+1)} x_{j}},
\end{array}\right.
$$

where $j \in\{0,1, \ldots, 2 p\},\left(L_{n}\right)_{n=0}^{+\infty}$ is the Lucas sequence and $\left(F_{n}\right)_{n=0}^{+\infty}$ is the Fibonacci sequence. 
Corollary 2. Let $\left\{x_{n}^{(1)}, x_{n}^{(2)}, \ldots, x_{n}^{(2 p+1)}\right\}_{n>0}$ be a well-defined solution of (2.1). Then, for $n \geq 2 p$

$$
\left\{\begin{array}{l}
x_{2(2 p+1) n+j}^{(q)}=\frac{F_{2(2 p+1) n+j}+x_{0}^{(q+j) \bmod (2 p+1)} F_{2(2 p+1) n+(j-1)}}{F_{2(2 p+1) n+(j+1)}+x_{0}^{(q+j) \bmod (2 p+1)} F_{2(2 p+1) n+j}} \\
x_{(2 p+1)(2 n+1)+j}^{(q)}=\frac{L_{(2 p+1)(2 n+1)+j}+x_{0}^{(q+j) \bmod (2 p+1)} L_{(2 p+1)(2 n+1)+(j-1)}}{L_{(2 p+1)(2 n+1)+(j+1)}+x_{0}^{(q+j) \bmod (2 p+1)} L_{(2 p+1)(2 n+1)+j}},
\end{array}\right.
$$

with $j \in\{0,2, \ldots, 2 p\}$.

$$
\left\{\begin{array}{l}
x_{2(2 p+1) n+j}^{(q)}=\frac{L_{2(2 p+1) n+(j+1)}+x_{0}^{(q+j) \bmod (2 p+1)} L_{2(2 p+1) n+j}}{L_{2(2 p+1) n+(j+2)}+x_{0}^{(q+j) \bmod (2 p+1)} L_{2(2 p+1) n+(j+1)}}, \\
x_{(2 p+1)(2 n+1)+j}^{(q)}=\frac{F_{(2 p+1)(2 n+1)+(j+1)}+x_{0}^{(q+j) \bmod (2 p+1)} F_{(2 p+1)(2 n+1)+j}}{F_{(2 p+1)(2 n+1)+(j+2)}+x_{0}^{(q+j) \bmod (2 p+1)} F_{(2 p+1)(2 n+1)+(j+1)}} .
\end{array}\right.
$$

with $j \in\{1,3, \ldots, 2 p+1\}, q \in\{1,2, \ldots, 2 p+1\},\left(L_{n}\right)_{n=0}^{+\infty}$ is the Lucas sequence and $\left(F_{n}\right)_{n=0}^{+\infty}$ is the Fibonacci sequence.

Proof. Let $\left\{x_{n}^{(1)}, x_{n}^{(2)}, \ldots, x_{n}^{(2 p+1)}\right\}_{n \geq 0}$ be a well-defined solution of system (2.1), so $\left\{x_{n}^{(1)}\right\}_{n \geq 0}$ is a solution of equation (2.2). Then,

$$
\begin{aligned}
x_{(2 p+1)(2 n)+j}^{(1)} & =\frac{F_{2(2 p+1) n}+F_{2(2 p+1) n-1} x_{j}^{(1)}}{F_{2(2 p+1) n+1}+F_{2(2 p+1) n} x_{j}^{(1)}}, \\
x_{(2 p+1)(2 n+1)+j}^{(1)} & =\frac{L_{(2 p+1)(2 n+1)}+L_{(2 p+1)(2 n+1)-1} x_{j}^{(1)}}{L_{(2 p+1)(2 n+1)+1}+L_{(2 p+1)(2 n+1)} x_{j}^{(1)}},
\end{aligned}
$$

$n \geq 2 p, j \in\{0,1, \ldots, 2 p\}$.

On the other hand, if $j$ is even, we have

$$
x_{j}^{(1)}=\frac{F_{j}+F_{j-1}{ }^{1} x_{0}^{(1+j)}}{F_{j+1}+F_{j}{ }^{1} x_{0}^{(1+j)}} .
$$

From (2.17) we get

$$
x_{(2 p+1)(2 n)+j}^{(1)}=\frac{F_{2(2 p+1) n}+F_{2(2 p+1) n-1} x_{j}^{(1)}}{F_{2(2 p+1) n+1}+F_{2(2 p+1) n} x_{j}^{(1)}} .
$$

Using (2.19) and the equalities

$$
F_{m}=F_{j+1} F_{m-j}+F_{j} F_{m-(j+1)}, \quad j \in 2 \mathbb{N}, m \in \mathbb{N},
$$


we obtain

$$
\begin{aligned}
x_{2(2 p+1) n+j}^{(1)}= & \frac{\left(F_{j+1}+F_{j} x_{0}^{(1+j)}\right) F_{2(2 p+1) n}+\left(F_{j}+F_{j-1} x_{0}^{(1+j)}\right) F_{2(2 p+1) n-1}}{\left(F_{j+1}+F_{j} x_{0}^{(1+j)}\right) F_{2(2 p+1) n+1}+\left(F_{j}+F_{j-1} x_{0}^{(1+j)}\right) F_{2(2 p+1) n}} \\
= & \frac{F_{2(2 p+1) n+j}+x_{0}^{(1+j)} F_{2(2 p+1) n+(j-1)}}{F_{2(2 p+1) n+(j+1)}+x_{0}^{(1+j)} F_{2(2 p+1) n+j}} .
\end{aligned}
$$

Similarly, from (2.17) we have

$$
x_{(2 p+1)(2 n+1)-j}^{(1)}=\frac{L_{(2 p+1)(2 n+1)}+L_{(2 p+1)(2 n+1)-1} x_{j}^{(1)}}{L_{(2 p+1)(2 n+1)+1}+L_{(2 p+1)(2 n+1)} x_{j}^{(1)}} .
$$

Using (2.19) and the (2.20) we obtain

$$
\begin{aligned}
x_{(2 p+1)(2 n+1)+j}^{(1)} & =\frac{\left(F_{j+1}+F_{j} x_{0}^{(1+j)}\right) L_{(2 p+1)(2 n+1)}+\left(F_{j}+F_{j-1} x_{0}^{(1+j)}\right) L_{(2 p+1)(2 n+1)-1}}{\left(F_{j+1}+F_{j} x_{0}^{(1+j)}\right) L_{(2 p+1)(2 n+1)+1}+\left(F_{j}+F_{j-1} x_{0}^{(1+j)}\right) L_{(2 p+1)(2 n+1)}} \\
& =\frac{L_{(2 p+1)(2 n+1)+j}+x_{0}^{(1+j)} L_{(2 p+1)(2 n+1)+(j-1)}}{L_{(2 p+1)(2 n+1)+(j+1)}+x_{0}^{(1+j)} L_{(2 p+1)(2 n+1)+j}} .
\end{aligned}
$$

If $j$ is odd, we have

$$
x_{j}^{(1)}=\frac{F_{j}+F_{j-1} x_{1}^{(j)}}{F_{j+1}+F_{j} x_{1}^{(j)}} .
$$

From (2.17) we have

$$
x_{(2 p+1)(2 n)+j}^{(1)}=\frac{F_{2(2 p+1) n}+F_{2(2 p+1) n-1} x_{j}^{(1)}}{F_{2(2 p+1) n+1}+F_{2(2 p+1) n} x_{j}^{(1)}} .
$$

From (2.20) and (2.21), we get

$$
\begin{aligned}
x_{2(2 p+1) n+j}^{(1)} & =\frac{\left(F_{j+1}+F_{j} x_{1}^{(j)}\right) F_{2(2 p+1) n}+\left(F_{j}+F_{j-1} x_{1}^{(j)}\right) F_{2(2 p+1) n-1}}{\left(F_{j+1}+F_{j} x_{1}^{(j)}\right) F_{2(2 p+1) n+1}+\left(F_{j}+F_{j-1} x_{1}^{(j)}\right) F_{2(2 p+1) n}} \\
= & \frac{F_{2(2 p+1) n+j}+x_{1}^{(j)} F_{2(2 p+1) n+(j-1)}}{F_{2(2 p+1) n+(j+1)}+x_{1}^{(j)} F_{2(2 p+1) n+j}}
\end{aligned}
$$

So,

$$
x_{(2 p+1)(2 n+1)+j}^{(1)}=\frac{L_{(2 p+1)(2 n+1)}+L_{(2 p+1)(2 n+1)-1} x_{j}^{(1)}}{L_{(2 p+1)(2 n+1)+1}+L_{(2 p+1)(2 n+1)} x_{j}^{(1)}} .
$$


From (2.21), we have

$$
\begin{aligned}
x_{(2 p+1)(2 n+1)+j}^{(1)} & =\frac{\left(F_{j+1}+F_{j} x_{1}^{(j)}\right) L_{(2 p+1)(2 n+1)}+\left(F_{j}+F_{j-1} x_{1}^{(j)}\right) L_{(2 p+1)(2 n+1)-1}}{\left(F_{j+1}+F_{j} x_{1}^{(j)}\right) L_{(2 p+1)(2 n+1)+1}+\left(F_{j}+F_{j-1} x_{1}^{(j)}\right) L_{(2 p+1)(2 n+1)}} \\
& =\frac{L_{(2 p+1)(2 n+1)+j}+x_{1}^{(j)} L_{(2 p+1)(2 n+1)+(j-1)}}{L_{(2 p+1)(2 n+1)+(j+1)}+x_{1}^{(j)} L_{(2 p+1)(2 n+1)+j}}
\end{aligned}
$$

So

$$
\left\{\begin{array}{l}
x_{2(2 p+1) n+j}^{(1)}=\frac{F_{2(2 p+1) n+j}+x_{1}^{(j)} F_{2(2 p+1) n+(j-1)}}{F_{2(2 p+1) n+(j+1)}+x_{1}^{(j)} F_{2(2 p+1) n+j}} \\
x_{(2 p+1)(2 n+1)-j}^{(1)}=\frac{L_{(2 p+1)(2 n+1)+j}+x_{1}^{(j)} L_{(2 p+1)(2 n+1)+(j-1)}}{L_{(2 p+1)(2 n+1)+(j+1)}+x_{1}^{(j)} L_{(2 p+1)(2 n+1)+j}} .
\end{array}\right.
$$

Since we have

$$
x_{1}^{(j)}=\frac{1+2 x_{0}^{(j+1)}}{3+x_{0}^{(j+1)}},
$$

we get

$$
\left\{\begin{array}{c}
x_{2(2 p+1) n+j}^{(1)}=\frac{F_{2(2 p+1) n+j}+\left(\frac{1+2 x_{0}^{(j+1)}}{3+x_{0}^{(j+1)}}\right) F_{2(2 p+1) n+(j-1)}}{F_{2(2 p+1) n+(j+1)}+\left(\frac{1+2 x_{0}^{(j+1)}}{3+x_{0}^{(j+1)}}\right) F_{2(2 p+1) n+j}} \\
x_{(2 p+1)(2 n+1)-j}^{(1)}=\frac{L_{(2 p+1)(2 n+1)+j}+\left(\frac{1+2 x_{0}^{(j+1)}}{3+x_{0}^{(j+1)}}\right) L_{(2 p+1)(2 n+1)+(j-1)}}{L_{(2 p+1)(2 n+1)+(j+1)}+\left(\frac{1+2 x_{0}^{(j+1)}}{3+x_{0}^{(j+1)}}\right) L_{(2 p+1)(2 n+1)+j}} .
\end{array}\right.
$$

So

$$
\left\{\begin{array}{l}
x_{2(2 p+1) n+j}^{(1)}=\frac{\left(3 F_{2(2 p+1) n+j}+F_{2(2 p+1) n+(j-1)}\right)+x_{0}^{(j+1)}\left(F_{2(2 p+1) n+j}+2 F_{2(2 p+1) n+(j-1)}\right)}{\left(3 F_{2(2 p+1) n+(j+1)}+F_{2(2 p+1) n+j}\right)+x_{0}^{(j+1)}\left(F_{2(2 p+1) n+(j+1)}+2 F_{2(2 p+1) n+j}\right)} \\
x_{(2 p+1)(2 n+1)+j}^{(1)} \\
\quad=\frac{\left(3 L_{(2 p+1)(2 n+1)+j}+L_{(2 p+1)(2 n+1)+(j-1)}\right)+x_{0}^{(j+1)}\left(2 L_{(2 p+1)(2 n+1)+(j-1)}+L_{(2 p+1)(2 n+1)+j}\right)}{\left(3 L_{(2 p+1)(2 n+1)+(j+1)}+L_{(2 p+1)(2 n+1)+j}\right)+x_{0}^{(j+1)}\left(2 L_{(2 p+1)(2 n+1)+j}+L_{(2 p+1)(2 n+1)+(j+1))}\right.}
\end{array}\right.
$$


Finally we get

Hence

$$
\left\{\begin{array}{l}
x_{2(2 p+1) n+j}^{(1)}=\frac{L_{2(2 p+1) n+(j+1)}+x_{0}^{(j+1)} L_{2(2 p+1) n+j}}{L_{2(2 p+1) n+(j+2)}+x_{0}^{(j+1)} L_{2(2 p+1) n+(j+1)}} \\
x_{(2 p+1)(2 n+1)+j}^{(1)}=\frac{F_{(2 p+1)(2 n+1)+(j+1)}+x_{0}^{(j+1)} F_{(2 p+1)(2 n+1)+j}}{F_{(2 p+1)(2 n+1)+(j+2)}+x_{0}^{(j+1)} F_{(2 p+1)(2 n+1)+(j+1)}} .
\end{array}\right.
$$

$$
\left\{\begin{array}{l}
x_{2(2 p+1) n+j}^{(1)}=\frac{F_{2(2 p+1) n+j}+x_{0}^{(1+j)} F_{2(2 p+1) n+(j-1)}}{F_{2(2 p+1) n+(j+1)}+x_{0}^{(1+j)} F_{2(2 p+1) n+j}} \\
x_{(2 p+1)(2 n+1)+j}^{(1)}=\frac{L_{(2 p+1)(2 n+1)+j}+x_{0}^{(1+j)} L_{(2 p+1)(2 n+1)+(j-1)}}{L_{(2 p+1)(2 n+1)+(j+1)}+x_{0}^{(1+j)} L_{(2 p+1)(2 n+1)+j}}
\end{array}\right.
$$

with $j \in\{0,2, \ldots, 2 p\}$.

$$
\left\{\begin{array}{l}
x_{2(2 p+1) n+j}^{(1)}=\frac{L_{2(2 p+1) n+(j+1)}+x_{0}^{(j+1)} L_{2(2 p+1) n+j}}{L_{2(2 p+1) n+(j+2)}+x_{0}^{(j+1)} L_{2(2 p+1) n+(j+1)}} \\
x_{(2 p+1)(2 n+1)+j}^{(1)}=\frac{F_{(2 p+1)(2 n+1)+(j+1)}+x_{0}^{(j+1)} F_{(2 p+1)(2 n+1)+j}}{F_{(2 p+1)(2 n+1)+(j+2)}+x_{0}^{(j+1)} F_{(2 p+1)(2 n+1)+(j+1)}},
\end{array}\right.
$$

with $j \in\{1,3, \ldots, 2 p+1\}$.

In the same way, after some calculations and using the fact that

$$
x_{n}^{(2 p+1)}=\frac{1+2 x_{n-1}^{(1)}}{3+x_{n-1}^{(1)}}, \quad x_{n}^{(i)}=\frac{1+2 x_{n-1}^{(i+1)}}{3+x_{n-1}^{(i+1)}}, \quad i=2,3, \ldots, 2 p,
$$

we obtain

$$
\left\{\begin{array}{l}
x_{2(2 p+1) n+j}^{(q)}=\frac{F_{2(2 p+1) n+j}+x_{0}^{(q+j) \bmod (2 p+1)} F_{2(2 p+1) n+(j-1)}}{F_{2(2 p+1) n+(j+1)}+x_{0}^{(q+j) \bmod (2 p+1)} F_{2(2 p+1) n+j}} \\
x_{(2 p+1)(2 n+1)+j}^{(q)}=\frac{L_{(2 p+1)(2 n+1)+j}+x_{0}^{(q+j) \bmod (2 p+1)} L_{(2 p+1)(2 n+1)+(j-1)}}{L_{(2 p+1)(2 n+1)+(j+1)}+x_{0}^{(q+j) \bmod (2 p+1)} L_{(2 p+1)(2 n+1)+j}},
\end{array}\right.
$$

with $j \in\{0,2, \ldots, 2 p\}$.

$$
\left\{\begin{array}{l}
x_{2(2 p+1) n+j}^{(q)}=\frac{L_{2(2 p+1) n+(j+1)}+x_{0}^{(q+j) \bmod (2 p+1)} L_{2(2 p+1) n+j}}{L_{2(2 p+1) n+(j+2)}+x_{0}^{(q+j) \bmod (2 p+1)} L_{2(2 p+1) n+(j+1)}}, \\
x_{(2 p+1)(2 n+1)+j}^{(q)}=\frac{F_{(2 p+1)(2 n+1)+(j+1)}+x_{0}^{(q+j) \bmod (2 p+1)} F_{(2 p+1)(2 n+1)+j}}{F_{(2 p+1)(2 n+1)+(j+2)}+x_{0}^{(q+j) \bmod (2 p+1)} F_{(2 p+1)(2 n+1)+(j+1)}},
\end{array}\right.
$$

with $j \in\{1,3, \ldots, 2 p+1\}$. 


\section{ON THE SYSTEM OF HIGHER ORDER DIFFERENCE EQUATIONS (1.9)}

In this section, we discuss the form of system (1.9) which generalizes (2.1) in a graceful way. We establish the solution of the system (1.9) by using an appropriate transformation reducing this system to the system of first-order difference equations (2.1).

\subsection{Analysis of the form of system (1.9)}

The initial values with the smallest indexes are $x_{-k}^{(1)}, x_{-k}^{(2)}, \ldots, x_{-k}^{(2 p)}$ and $x_{-k}^{(2 p+1)}$. By using (1.9) with $n=0$, we obtain the values of $x_{1}^{(1)}, x_{1}^{(2)}, \ldots, x_{1}^{(2 p)}$ and $x_{1}^{(2 p+1)}$ as follows

$$
x_{1}^{(1)}=\frac{1+2 x_{-k}^{(1)}}{3+x_{-k}^{(1)}}, \quad x_{1}^{(2)}=\frac{1+2 x_{-k}^{(3)}}{3+x_{-k}^{(3)}}, \cdots, \quad x_{1}^{(2 p+1)}=\frac{1+2 x_{-k}^{(1)}}{3+x_{-k}^{(1)}} .
$$

After known the values of $x_{1}^{(1)}, x_{1}^{(2)}, \ldots, x_{1}^{(2 p)}$ and $x_{1}^{(2 p+1)}$, by using (1.9) with $n=k+1$ we get the values of $x_{k+2}^{(1)}, x_{k+2}^{(2)}, \ldots, x_{k+2}^{(2 p)}$ and $x_{k+2}^{(2 p+1)}$. We have

$$
x_{k+2}^{(1)}=\frac{1+2 x_{1}^{(1)}}{3+x_{1}^{(1)}}, \quad x_{k+2}^{(2)}=\frac{1+2 x_{1}^{(3)}}{3+x_{1}^{(3)}}, \cdots, \quad x_{k+2}^{(2 p+1)}=\frac{1+2 x_{1}^{(1)}}{3+x_{1}^{(1)}} .
$$

The values of $x_{k+2}^{(1)}, x_{k+2}^{(2)}, \ldots, x_{k+2}^{(2 p)}$ and $x_{k+2}^{(2 p+1)}$, by using (1.9) with $n=2 k+2$, leads us to obtain the values of $x_{2 k+3}^{(1)}, x_{2 k+3}^{(2)}, \ldots, x_{2 k+3}^{(2 p)}$ and $x_{2 k+3}^{(2 p+1)}$. We have

$$
\begin{aligned}
& x_{2 k+3}^{(1)}=\frac{1+2 x_{k+2}^{(1)}}{3+x_{k+2}^{(1)}}, \quad x_{2 k+3}^{(2)}=\frac{1+2 x_{k+2}^{(3)}}{3+x_{k+2}^{(3)}}, \cdots, \quad x_{2 k+3}^{(2 p+1)}=\frac{1+2 x_{k+2}^{(1)}}{3+x_{k+2}^{(1)}} . \\
& \left\{\begin{aligned}
x_{(k+1) m+1}^{(1)} & =\frac{1+2 x_{(k+1) m-k}^{(1)}}{3+x_{(k+1) m-k}^{(1)}}, \\
x_{(k+1) m+1}^{(2)} & =\frac{1+2 x_{(k+1) m-k}^{(3)}}{3+x_{(k+1) m-k}^{(3)}}, \\
& \vdots \\
x_{(k+1) m+1}^{(2 p+1)} & =\frac{1+2 x_{(k+1) m-k}^{(1)}}{3+x_{(k+1) m-k}^{(1)}} .
\end{aligned}\right.
\end{aligned}
$$

In the same way, it is shown that the initial values $x_{-r}^{(1)}, x_{-r}^{(2)}, \ldots, x_{-r}^{(2 p)}$ and $x_{-r}^{(2 p+1)}$, for a fixed $r \in\{0,1, \ldots, k\}$, determine all the values of the sequences $\left(x_{(k+1)(m+1)-r}^{(1)}\right)_{m}$, 
$\left(x_{(k+1)(m+1)-r}^{(2)}\right)_{m}, \ldots,\left(x_{(k+1)(m+1)-r}^{(2 p)}\right)_{m}$ and $\left(x_{(k+1)(m+1)-r}^{(2 p+1)}\right)_{m}$. Also we have

$$
\left\{\begin{aligned}
x_{(k+1)(m+1)-r}^{(1)} & =\frac{1+2 x_{(k+1) m-r}^{(1)}}{3+x_{(k+1) m-r}^{(1)}} \\
x_{(k+1)(m+1)-r}^{(2)} & =\frac{1+2 x_{(k+1) m-r}^{(3)}}{3+x_{(k+1) m-r}^{(3)}} \\
& \vdots \\
x_{(k+1)(m+1)-r}^{(2 p+1)} & =\frac{1+2 x_{(k+1) m-r}^{(1)}}{3+x_{(k+1) m-r}^{(1)}} .
\end{aligned}\right.
$$

\subsection{A representation of the general solution to system (1.9)}

Now we are going to apply the previous analysis. Let

$$
{ }^{(r)} x_{n}^{(q)}=x_{(k+1) n-r},
$$

where $r \in\{0,1, \ldots, k\}$. and $q \in\{1,2, \ldots,(2 p+1)\}$.

Using notation (3.3), we can write (1.9) as

$$
{ }^{(r)} x_{n+1}^{(1)}=\frac{1+2^{(r)} x_{n}^{(1)}}{3+{ }^{(r)} x_{n}^{(1)}}, \quad{ }^{(r)} x_{n+1}^{(2)}=\frac{1+2^{(r)} x_{n}^{(3)}}{3+{ }^{(r)} x_{n}^{(3)}}, \cdots, \quad{ }^{(r)} x_{n+1}^{(2 p+1)}=\frac{1+2^{(r)} x_{n}^{(1)}}{3+{ }^{(r)} x_{n}^{(1)}},
$$

for each $r \in\{0,1, \ldots, k\}$.

It signifies that the sequences $\left({ }^{(r)} x_{n}^{(1)}\right)_{n \in \mathbb{N}_{0}},\left({ }^{(r)} x_{n}^{(2)}\right)_{n \in \mathbb{N}_{0}}, \ldots,\left({ }^{(r)} x_{n}^{(2 p)}\right)_{n \in \mathbb{N}_{0}}$ and $\left({ }^{(r)} x_{n}^{(2 p+1)}\right)_{n \in \mathbb{N}_{0}}, r=\overline{0, k}$, are $(2 p+1)(k+1)$ solutions to system (2.1) with the initial values ${ }^{(r)} x_{0}^{(1)},{ }^{(r)} x_{0}^{(2)}, \ldots,{ }^{(r)} x_{0}^{(2 p)}$ and ${ }^{(r)} x_{0}^{(2 p+1)}, r=\overline{0, k}$, respectively.

Using Corollary (2) to the sequences $\left({ }^{(r)} x_{n}^{(1)}\right)_{n \in \mathbb{N}_{0}},\left({ }^{(r)} x_{n}^{(2)}\right)_{n \in \mathbb{N}_{0}}, \ldots,\left({ }^{(r)} x_{n}^{(2 p)}\right)_{n \in \mathbb{N}_{0}}$ and $\left({ }^{(r)} x_{n}^{(2 p+1)}\right)_{n \in \mathbb{N}_{0}}, r=\overline{0, k}$, we show that the following representation holds

$$
\left\{\begin{array}{l}
{ }^{(r)} x_{2(2 p+1) n+j}^{(q)}=\frac{F_{2(2 p+1) n+j}{ }^{(r)}+x_{0}^{(q+j) \bmod (2 p+1)} F_{2(2 p+1) n+(j-1)}}{F_{2(2 p+1) n+(j+1)}+{ }^{(r)} x_{0}^{(q+j) \bmod (2 p+1)} F_{2(2 p+1) n+j}} \\
{ }^{(r)} x_{(2 p+1)(2 n+1)+j}^{(q)}=\frac{L_{(2 p+1)(2 n+1)+j}+{ }^{(r)} x_{0}^{(q+j) \bmod (2 p+1)} L_{(2 p+1)(2 n+1)+(j-1)}}{L_{(2 p+1)(2 n+1)+(j+1)}+{ }^{(r)} x_{0}^{(q+j) \bmod (2 p+1)} L_{(2 p+1)(2 n+1)+j}},
\end{array}\right.
$$


with $j \in\{0,2, \ldots, 2 p\}$.

$$
\left\{\begin{array}{l}
{ }^{(r)} x_{2(2 p+1) n+j}^{(q)}=\frac{L_{2(2 p+1) n+(j+1)}+{ }^{(r)} x_{0}^{(q+j) \bmod (2 p+1)} L_{2(2 p+1) n+j}}{L_{2(2 p+1) n+(j+2)}+{ }^{(r)} x_{0}^{(q+j) \bmod (2 p+1)} L_{2(2 p+1) n+(j+1)}}, \\
{ }^{(r)} x_{(2 p+1)(2 n+1)+j}^{(q)}=\frac{F_{(2 p+1)(2 n+1)+(j+1)}+{ }^{(r)} x_{0}^{(q+j) \bmod (2 p+1)} F_{(2 p+1)(2 n+1)+j}}{F_{(2 p+1)(2 n+1)+(j+2)}+{ }^{(r)} x_{0}^{(q+j) \bmod (2 p+1)} F_{(2 p+1)(2 n+1)+(j+1)}},
\end{array}\right.
$$

with $j \in\{1,3, \ldots, 2 p+1\}$.

For each $q \in\{1,2, \ldots, 2 p+1\}, r \in\{1,2, \ldots, k\},\left(L_{n}\right)_{n=0}^{+\infty}$ is the Lucas sequence and $\left(F_{n}\right)_{n=0}^{+\infty}$ is the Fibonacci sequence.

Coming back to the original notation, from (3.3), it follows that the following result holds.

Corollary 3. Let $\left\{x_{n}^{(1)}, x_{n}^{(2)}, \ldots, x_{n}^{(2 p+1)}\right\}_{n \geq-k}$ be a solution of (1.9). Then, for $n=2,3, \ldots$,

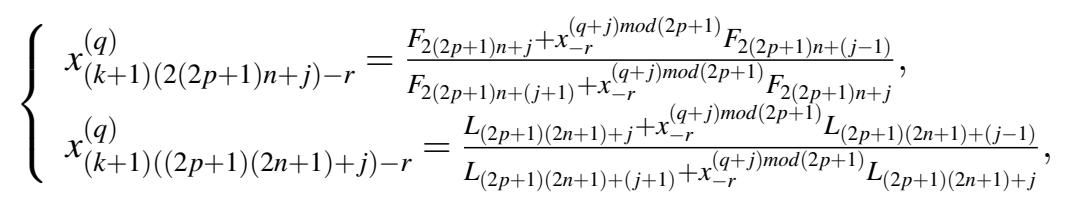

with $j \in\{0,2, \ldots, 2 p\}$.

$$
\left\{\begin{array}{l}
x_{(k+1)(2(2 p+1) n+j)-r}^{(q)}=\frac{L_{2(2 p+1) n+(j+1)}+x_{-r}^{(q+j) m o d(2 p+1)} L_{2(2 p+1) n+j}}{L_{2(2 p+1) n+(j+2)}+x_{-r}^{(+j) m o d(2 p+1)} L_{2(2 p+1) n+(j+1)}} \\
x_{(k+1)((2 p+1)(2 n+1)+j)-r}^{(q)}=\frac{F_{(2 p+1)(2 n+1)+(j+1)}+x_{-r}^{(q+j) \bmod (2 p+1)} F_{(2 p+1)(2 n+1)+j}}{F_{(2 p+1)(2 n+1)+(j+2)}+x_{-r}^{(q+j) m o d(2 p+1)} F_{(2 p+1)(2 n+1)+(j+1)}},
\end{array}\right.
$$

where $j \in\{1,3, \ldots, 2 p+1\}, q \in\{1,2, \ldots, 2 p+1\}, r \in\{1,2, \ldots, k\},\left(L_{n}\right)_{n=0}^{+\infty}$ is the Lucas sequence and $\left(F_{n}\right)_{n=0}^{+\infty}$ is the Fibonacci sequence.

\section{Global Stability of Positive solutions of (1.9)}

In this section we study the global stability character of the solutions of system (1.9). It is easy to show that (1.9) has a unique real positive equilibrium point given by

$$
E=\left(\overline{x^{(1)}}, \overline{x^{(2)}}, \ldots, \overline{x^{(2 p+1)}}\right)=(-\beta,-\beta, \ldots,-\beta),
$$

where $\beta$ is the number defined in (1.3).

Let $I_{i}(0,+\infty)$ and consider the functions

$$
f_{i}: I_{1}^{k+1} \times I_{2}^{k+1} \times \ldots \times I_{2 p+1}^{k+1} \longrightarrow I_{i}
$$

defined by 


$$
\begin{aligned}
f_{i}\left(u_{0}^{(1)}, u_{1}^{(1)}, \ldots, u_{k}^{(1)}, u_{0}^{(2)}, u_{1}^{(2)}, \ldots, u_{k}^{(2)}, \ldots, u_{0}^{(2 p+1)}, u_{1}^{(2 p+1)}, \ldots, u_{k}^{(2 p+1)}\right) \\
=\frac{1+2 u_{k}^{(i+1) \bmod (2 p+1)}}{3+u_{k}^{(i+1) \bmod (2 p+1)}},
\end{aligned}
$$

with $i \in\{1,2, \ldots, 2 p+1\}$.

Theorem 2. The equilibrium point $E$ is locally asymptotically stable.

Proof. The linearized system about the equilibrium point

$$
E=(-\beta, \ldots,-\beta,-\beta, \ldots,-\beta) \in I_{1}^{k+1} \times I_{2}^{k+1} \times \ldots \times I_{2 p+1}^{k+1}
$$

is given by

$$
X_{n+1}=A X_{n}
$$

$$
X_{n}=\left(x_{n}^{(1)}, x_{n-1}^{(1)}, \ldots, x_{n-k}^{(1)}, x_{n}^{(2)}, x_{n-1}^{(2)}, \ldots, x_{n-k}^{(2)}, \ldots, x_{n}^{(2 p+1)}, x_{n-1}^{(2 p+1)}, \ldots, x_{n-k}^{(2 p+1)}\right)^{t}
$$

and

$$
A=\left(\begin{array}{cccccccccccccc}
0 & 0 & \ldots & 0 & 0 & \ldots & 0 & \frac{5}{(3-\beta)^{2}} & \ldots & \ldots & 0 & 0 & \ldots & 0 \\
1 & 0 & & & 0 & & & & & & & & & 0 \\
0 & 1 & \ddots & & & & & & & & & & & \vdots \\
0 & & \ddots & 0 & \ldots & \ldots & 0 & 0 & \ldots & \ldots & 0 & 0 & \ldots & 0 \\
& & & 1 & 0 & & & & & & & & & \vdots \\
\vdots & & \vdots & & \vdots & & & \vdots & & & \vdots & & & \vdots \\
& & & & & 1 & & & & & & & & 0 \\
\vdots & & & \vdots & & 0 & & 1 & & & & & & 0 \\
\vdots & & & & & & & & & & & & & \vdots \\
0 & \ldots & 0 & 5 & 0 & 0 & & & \ldots & \ldots & 0 & & & \\
0 & & & 0 & & 0 & & & & 0 & \ddots & 0 & \vdots & \vdots \\
\vdots & & & \vdots & & \vdots & & & & & & \ddots & 0 & 0 \\
0 & 0 & \ldots & 0 & & 0 & 0 & \ldots & \ldots & \ldots & 0 & 0 & 1 & 0
\end{array}\right) .
$$

So, after some elementary calculations, we get

$$
P(\lambda)=(-\lambda)^{(2 p+1)(k+1)}+(-1)^{k}\left(\frac{5}{(3-\beta)^{2}}\right)^{2 p+1} .
$$


Now, consider the two functions defined by

$$
\varphi(\lambda)=\lambda^{(2 p+1)(k+1)}, \quad \phi(\lambda)=\left(\frac{5}{(3-\beta)^{2}}\right)^{2 p+1} .
$$

We have

$$
|\phi(\lambda)|<|\varphi(\lambda)|, \forall \lambda:|\lambda|=1
$$

So, according to Rouche's Theorem $\varphi$ and $P=\varphi+\phi$ have the same number of zeros in the unit disc $|\lambda|<1$, and since $\varphi$ admits as root $\lambda=0$ of multiplicity $(2 p+1)(k+1)$, then all the roots of $\mathrm{P}$ are in the disc $|\lambda|<1$. Thus, the equilibrium point is locally asymptotically stable.

Theorem 3. For every well defined solution of system (1.9), we have

$$
\lim _{n \rightarrow+\infty} x_{n}^{(q)}=-\beta
$$

for each $q \in\{1,2, \ldots, 2 p+1\}$.

Proof. From Corollary (3), we have

$$
\begin{aligned}
\lim _{n \rightarrow+\infty} x_{(k+1)(2(2 p+1) n+2(2 p+1)+j)-r}^{(q)} & \\
= & \lim _{n \rightarrow+\infty} \frac{F_{2(2 p+1) n+2(2 p+1)+j}+x_{-r}^{(q+j)} F_{2(2 p+1) n+2(2 p+1)+(j-1)}}{F_{2(2 p+1) n+2(2 p+1)+(j+1)-r}+x_{-r}^{(q+j)} F_{2(2 p+1) n+2(2 p+1)+j}} \\
& =\lim _{n \rightarrow+\infty} \frac{1+x_{-r}^{(q+j)} \frac{F_{2(2 p+1) n+2(2 p+1)+(j-1)}}{F_{2(2 p+1) n+2(2 p+1)+j}}}{\frac{F_{2(2 p+1) n+2(2 p+1)+(j+1)}+x_{-r}^{(q+j)}}{F_{2(2 p+1) n+2(2 p+1)+j}}} .
\end{aligned}
$$

Using the limit (1.4), we get

$$
\lim _{n \rightarrow+\infty} x_{(k+1)(2(2 p+1) n+2(2 p+1)+j)-r}^{(q)}=\frac{1+x_{-r}^{(q+j)} \frac{1}{\alpha}}{\alpha+x_{-r}^{(q+j)}} .
$$

Hence

$$
\lim _{n \rightarrow+\infty} x_{(k+1)(2(2 p+1) n+2(2 p+1)+j)-r}^{(q)}=-\beta .
$$

Also,

$$
\begin{aligned}
& \lim _{n \rightarrow+\infty} x_{(k+1)((2 p+1)(2 n+1)+j)-r}^{(q)} \\
&=\lim _{n \rightarrow+\infty} \frac{L_{2(2 p+1) n+(2 p+1)+j}+x_{-r}^{(q+j)} L_{2(2 p+1) n+(2 p+1)+(j-1)}}{L_{2(2 p+1) n+(2 p+1)+(j+1)}+x_{-r}^{(q+j)} L_{2(2 p+1) n+(2 p+1)+j}} \\
&=\lim _{n \rightarrow+\infty} \frac{1+x_{-r}^{(q+j)} \frac{L_{2(2 p+1) n+(2 p+1)+(j-1)}}{L_{2(2 p+1) n+(2 p+1)+j}}}{\frac{L_{2(2 p+1) n+(2 p+1)+(j+1)}+x_{-r}^{(q+j)}}{L_{2(2 p+1) n+(2 p+1)+j}}} .
\end{aligned}
$$


Using the limit (1.7), we get

$$
\lim _{n \rightarrow+\infty} x_{(k+1)((2 p+1)(2 n+1)+j)-r}^{(q)}=\frac{1+x_{-r}^{(q+j)} \frac{1}{\alpha}}{\alpha+x_{-r}^{(q+j)}} .
$$

Hence

Similarly, we find

$$
\lim _{n \rightarrow+\infty} x_{(k+1)((2 p+1)(2 n+1)+j)-r}^{(q)}=-\beta .
$$

$$
\lim _{n \rightarrow+\infty} x_{(k+1)(2(2 p+1) n+2(2 p+1)+j)-r}^{(q)}=\lim _{n \rightarrow+\infty} x_{(k+1)((2 p+1)(2 n+1)+j)-r}^{(q)}=-\beta .
$$

So, we have

$$
\lim _{n \rightarrow+\infty} x_{n}^{(q)}=-\beta
$$

The following result is a direct consequence of Theorems (2) and (3).

Corollary 4. The equilibrium point $E$ is globally asymptotically stable.

\subsection{Numerical confirmation}

In order to verify our theoretical results we consider several interesting numerical examples in this section. These examples represent different types of qualitative behaviour of solutions of the system (1.9). All plots in this section are drawn with Matlab.

Example 1. Let $k=1$ and $p=2$ in system (1.9), then we obtain the system

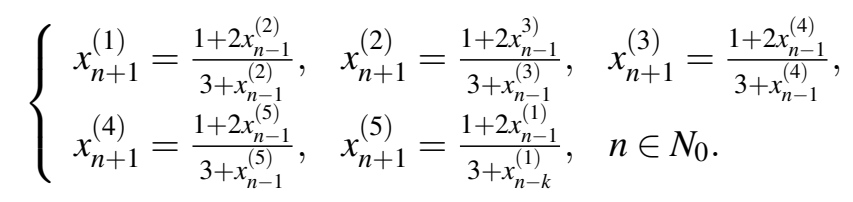

Assume $x_{-1}^{(1)}=1, x_{0}^{(1)}=7, x_{-1}^{(2)}=1.3, x_{0}^{(2)}=0.3, x_{-1}^{(3)}=3, x_{0}^{(3)}=1.5, x_{-1}^{(4)}=14$, $x_{0}^{(4)}=2, x_{-1}^{(5)}=3$ and $x_{0}^{(5)}=0.1$. (See Figure (1)).

Example 2. Let $k=3$ and $p=3$ in system (1.9), then we obtain the system

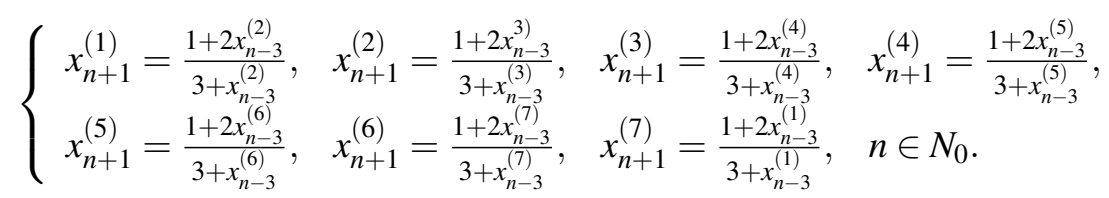

Assume $x_{-3}^{(1)}=1, x_{-2}^{(1)}=0.2, x_{-1}^{(1)}=6, x_{0}^{(1)}=7, x_{-3}^{(2)}=1.3, x_{-2}^{(2)}=5, x_{-1}^{(2)}=0.7, x_{0}^{(2)}=$ $9, x_{-3}^{(3)}=0.1, x_{-2}^{(3)}=3, x_{-1}^{(3)}=6, x_{0}^{(3)}=1.5, x_{-3}^{(4)}=7, x_{-2}^{(4)}=9.3, x_{-1}^{(4)}=5.3, x_{0}^{(4)}=5.3$, $x_{-3}^{(5)}=2.2, x_{-2}^{(5)}=2.2, x_{-1}^{(5)}=14.3, x_{0}^{(5)}=0.8, x_{-3}^{(6)}=3.3, x_{-2}^{(6)}=6, x_{-1}^{(6)}=8, x_{0}^{(6)}=1.9$, $x_{-3}^{(7)}=4, x_{-2}^{(7)}=7.2, x_{-1}^{(7)}=1.6$ and $x_{0}^{(7)}=8$. ( See Figure $(2)$ ). 


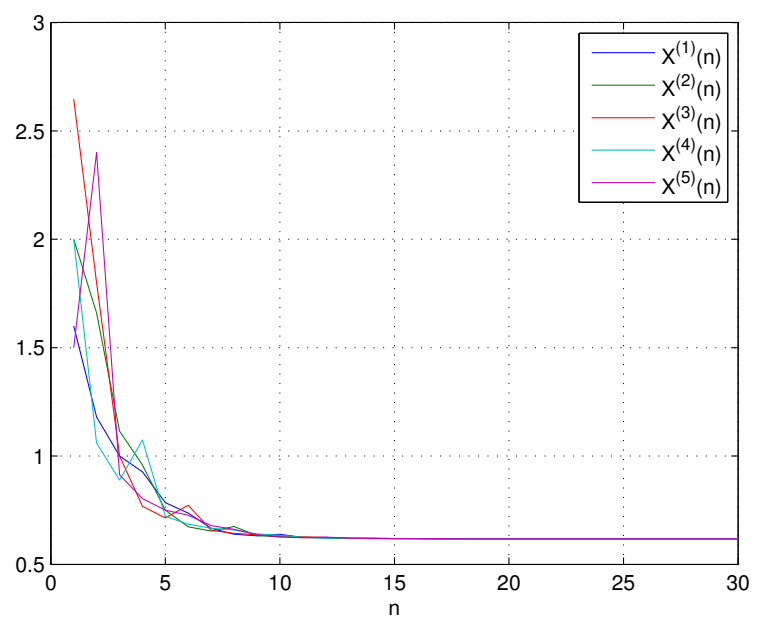

FIGURE 1. The plot of system (4.3)

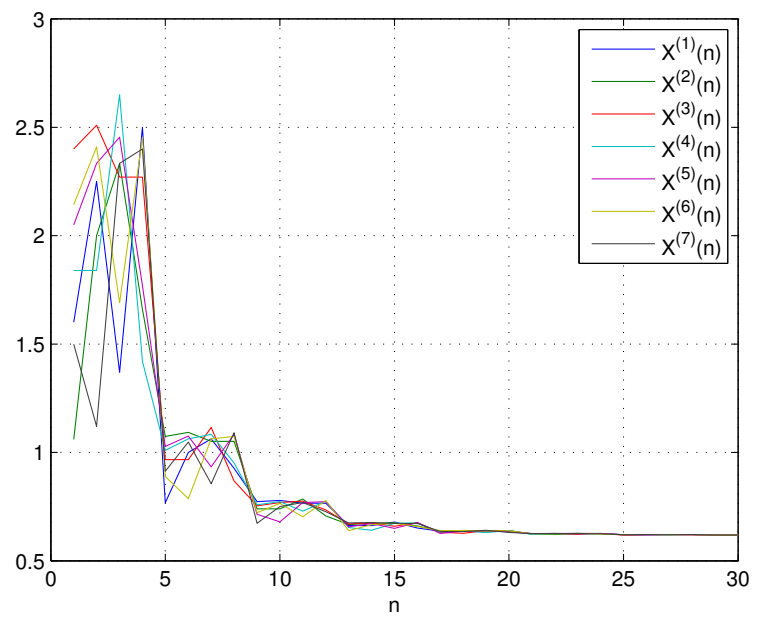

FIGURE 2. The plot of system (4.4)

\section{CONCLUSIONS}

In the paper, we represented the well-defined solutions of the system (1.9) composed by $2 p+1$ rational difference equations. More exactly, We gave general solutions of system (1.9) in terms of Fibonacci and Lucas sequences. Also, we presented 
some results about the general behavior of solutions of system (1.9) and some numerical examples are carried out to support the analysis results. Our system generalized the systems studied in [18] and [19].

The results in this paper can be extended to the following system of difference equations

$$
x_{n+1}^{(j)}=\frac{L_{m+2}+L_{m+1} x_{n-k}^{((j+1) \bmod (p))}}{L_{m+3}+L_{m+2} x_{n-k}^{((j+1) \bmod (p))}}, \quad n, m, p, k \in N_{0}, j=\overline{1, p},
$$

where $\left(L_{n}\right)_{n=0}^{+\infty}$ is the Lucas sequence.

\section{ACKNOWLEDGMENTS}

The authors would like to thank the anonymous referee for his/her remarks and suggestions.

\section{REFERENCES}

[1] R. Abo-Zeid, "Global behavior of a higher order difference equation," Math. Slovaca, vol. 64, no. 4, pp. 931-940, 2014, doi: 10.2478/s12175-014-0249-z.

[2] R. Abo-Zeid, "Forbidden sets and stability in some rational difference equations," J. Difference Equ. Appl., vol. 24, no. 2, pp. 220-239, 2018, doi: 10.1080/10236198.2017.1400023.

[3] Y. Akrour, N. Touafek, and Y. Halim, "On a system of difference equations of second order solved in closed-form." Miskolc Math. Notes, vol. 20, no. 2, pp. 701-717, 2019, doi: 10.18514/MMN.2019.2923.

[4] S. Elaydi, An introduction to difference equations. Springer Science \& Business Media, 2005. doi: 10.1007/0-387-27602-5.

[5] E. M. Elsayed, "Solutions of rational difference systems of order two," Math. Comput. Modelling, vol. 55, no. 1, pp. 378-384, 2012, doi: 10.1016/j.mcm.2011.08.012.

[6] E. M. Elsayed, "Solution for systems of difference equations of rational form of order two," Comp. Appl. Math., vol. 33, no. 3, pp. 751-765, 2014, doi: 10.1007/s40314-013-0092-9.

[7] E. M. Elsayed and T. F. Ibrahim, "Periodicity and solutions for some systems of nonlinear rational difference equations," Hacet. J. Math. Stat., vol. 44, no. 6, pp. 1361-1390, 2015, doi: 10.15672/HJMS.2015449653.

[8] E. A. Grove and G. Ladas, Periodicities in nonlinear difference equations. CRC Press, 2004, vol. 4.

[9] Y. Halim, "Form and periodicity of solutions of some systems of higher-order difference equations," Math. Sci. Lett., vol. 5, no. 1, pp. 79-84, 2016, doi: 10.18576/msl/050111.

[10] Y. Halim, "A system of difference equations with solutions associated to Fibonacci numbers," Int. J. Difference. Equ., vol. 11, no. 1, pp. 65-77, 2016.

[11] Y. Halim, "Global character of systems of rational difference equations," Electron. J. Math. Analysis. Appl., vol. 3, no. 1, pp. 204-214, 2018.

[12] Y. Halim and M. Bayram, "On the solutions of a higher-order difference equation in terms of generalized Fibonacci sequences," Math. Methods Appl. Sci., vol. 39, no. 1, pp. 2974-2982, 2016, doi: 10.1002/mma.3745.

[13] Y. Halim, A. Khelifa, and M. Berkal, "Representation of solutions of a two-dimensional System of difference equations," Miskolc Math. Notes, vol. 21, no. 1, pp. 203-2018, 2020, doi: 10.18514/MMN.2020.3204. 
[14] Y. Halim, A. Khelifa, and A. Boussaha, "Representation of solutions of a second-order system of difference equations in terms of Padovan sequence," Dyn. Contin. Discrete Impuls. Syst., Ser. B, Appl. Algorithms, vol. 27, no. 3, pp. 113-131, 2020.

[15] Y. Halim and J. F. T. Rabago, "On the solutions of a second-order difference equation in terms of generalized Padovan sequences," Math. Slovaca, vol. 68, no. 3, pp. 625-638, 2018, doi: 10.1515/ms-2017-0130.

[16] M. Kara and Y. Yazlik, "Solvability of a system of nonlinear difference equations of higher order," Turk. J. Math., vol. 43, no. 3, pp. 1533-1565, 2019, doi: 10.3906/mat-1902-24.

[17] W. G. Kelley and A. C. Peterson, Difference equations: an introduction with applications. Academic press, 2001.

[18] A. Khelifa, Y. Halim, and M. Berkal, "Solutions of a system of two higher-order difference equations in terms of Lucas sequence," Univers. J. Math. Appl., vol. 2, no. 4, pp. 202-211, 2019, doi: 10.32323/ujma.610399.

[19] A. Khelifa, Y. Halim, A. Bouchair, and M. Berkal, "On a system of three difference equations of higher order solved in terms of lucas and fibonacci numbers," Math. Slovaca, vol. 70, no. 3, pp. 641-656, 2020, doi: 10.1515/ms-2017-0378.

[20] T. Koshy, Fibonacci and Lucas numbers with applications. John Wiley \& Sons, 2019. doi: 10.1002/9781118033067.

[21] D. T. Tollu, Y. Yazlik, and N. Taskara, "On the solutions of two special types of Riccati difference equation via Fibonacci numbers," Adv. Difference Equ., vol. 174, no. 1, pp. 1-7, 2013, doi: 10.1186/1687-1847-2013-174.

[22] N. Touafek, "On some fractional systems of difference equations," Iran. J. Math. Sci. Inform., vol. 9, no. 2, pp. 73-86, 2014, doi: 10.7508/ijmsi.2014.02.007.

[23] N. Touafek and E. Elsayed, "On the solutions of systems of rational difference equations," Math. Comput. Modelling, vol. 55, no. 7-8, pp. 1987-1997, 2012, doi: 10.1016/j.mcm.2011.11.058.

Authors' addresses

A. Khelifa

Amira Khelifa, University of Mohamed Seddik Ben Yahia, LMAM Laboratory and Department of Mathematics, Jijel, Algeria

E-mail address: amkhelifa@yahoo.com

Y. Halim

Yacine Halim, Abdelhafid Boussouf University of Mila, Department of Mathematics and Computer Science, and LMAM Laboratory, University of Mohamed Seddik Ben Yahia, Jijel, Algeria

E-mail address: halyacine@yahoo.fr

M. Berkal

Messaoud Berkal, University of Alicante, Department of Applied Mathematics, Alicante, Spain

E-mail address: mb299@gcluad.ua.es 